\title{
Brain imaging in neonatal hypoglycaemia
}

\author{
Zoë Traill, Marian Squier, Philip Anslow
}

\begin{abstract}
Magnetic resonance imaging studies in two cases of neonatal hypoglycaemia showed cortical and white matter cerebral damage that was most obvious in the occipital lobes. Both cases showed oedema in the parieto-occipital cortex and underlying white matter in the acute phase, with profound atrophy of these regions in the chronic phase.
\end{abstract}

These findings support those of pathological studies which suggest that hypoglycaemia induces cerebral damage by a mechanism separate from the effects of cerebral hypoxia-ischaemia caused by secondary seizures.

(Arch Dis Child Fetal Neonatal Ed 1998;79:F145-F147)

Keywords: magnetic resonance imaging; computed tomography; hypoglycaemia; cerebral damage

Previous authors have considered it impossible to differentiate the cerebral damage associated with neonatal hypoglycaemia from that of associated hypoxia-ischaemia on imaging studies. ${ }^{1}$ However, a recent report described a case of neonatal hypoglycaemia in which imaging studies revealed extensive occipital cortical loss, ${ }^{2}$ a finding which correlated with previously published pathological studies. ${ }^{34}$ We

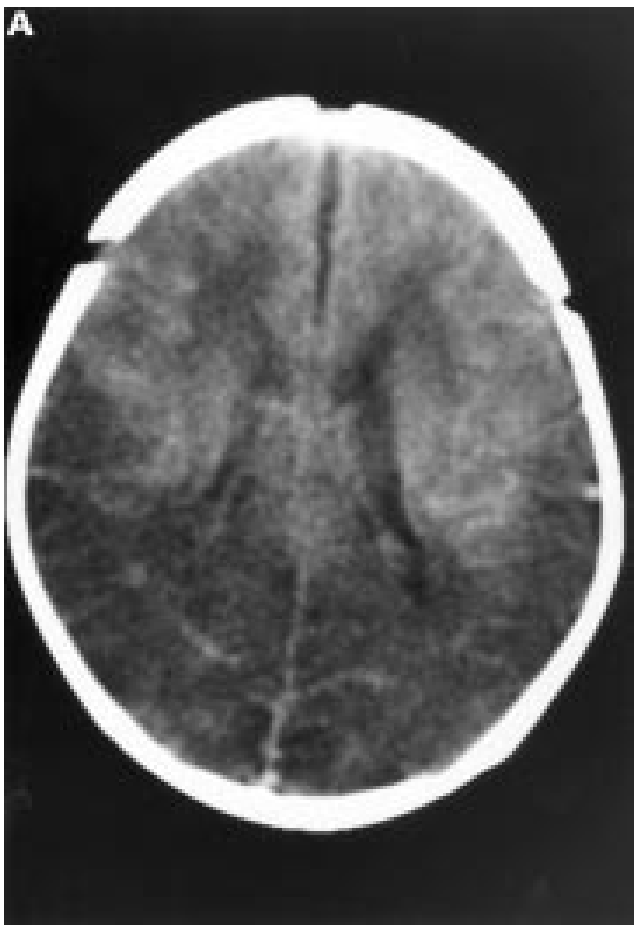

Figure 1A Case 1. Boy with isolated hypoglycaemia: computed tomography at 6 days of age shows cortical and white matter low density that is most severe in the parietal and occipital lobes. report two further cases of neonatal hypoglycaemia in which the most severe involvement occurred in the occipital lobes bilaterally, suggesting that imaging studies can differentiate between cerebral injury caused by hypoglycaemia and that resulting from hypoxiaischaemia.

\section{Case reports}

CASE 1

A boy was delivered to a non-diabetic primigravida at term. Both pregnancy and delivery were uncomplicated; birthweight was $3.4 \mathrm{~kg}$. Apgar scores were 8 at one minute and 9 at five minutes and there was no need for resuscitation. The infant fed well initially and was discharged from hospital at 24 hours. About two days after delivery the parents noted that he was feeding poorly and over the next 12 hours he became increasingly jittery and sleepy. At $2 \frac{1}{2}$ days of age he was admitted to hospital with seizures; his serum glucose measured $0.8 \mathrm{mmol} / 1$ (normal range $4.2-6.1 \mathrm{mmol} /$ 1). Intravenous dextrose was started but despite this the infant had recurrent episodes of hypoglycaemia and seizures over the next three days. A computed tomography scan performed at 6 days showed low density in the cortex and white matter throughout the brain, but with predominant involvement of the parietal and

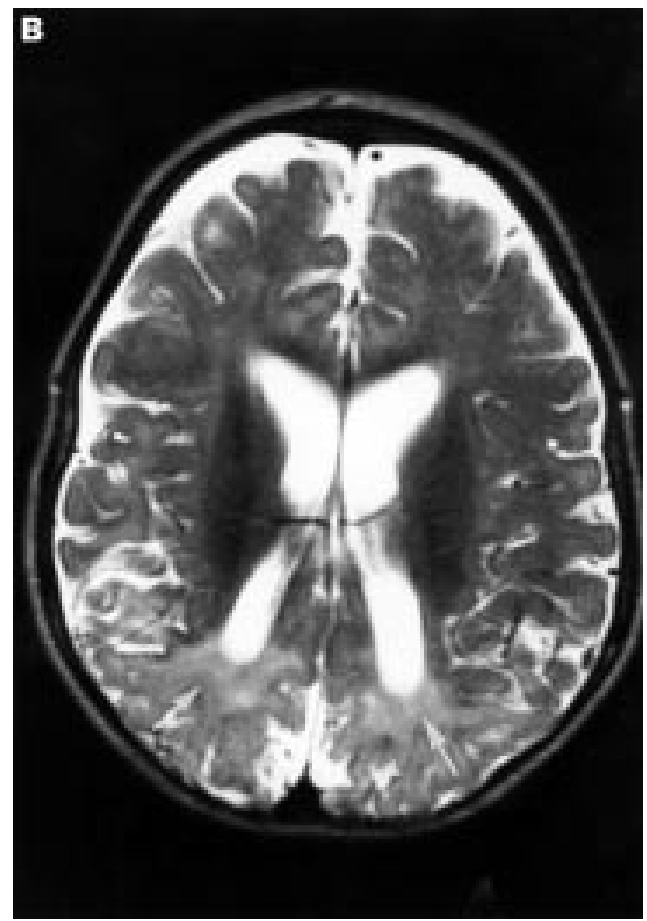

Figure $1 B \quad T_{2}$ weighted axial MRI at 10 months of age shows parenchymal loss posteriorly with high signal in the white matter of the parietal and occipital lobes (arrows). Note thin and atrophic gyri (arrowhead). 


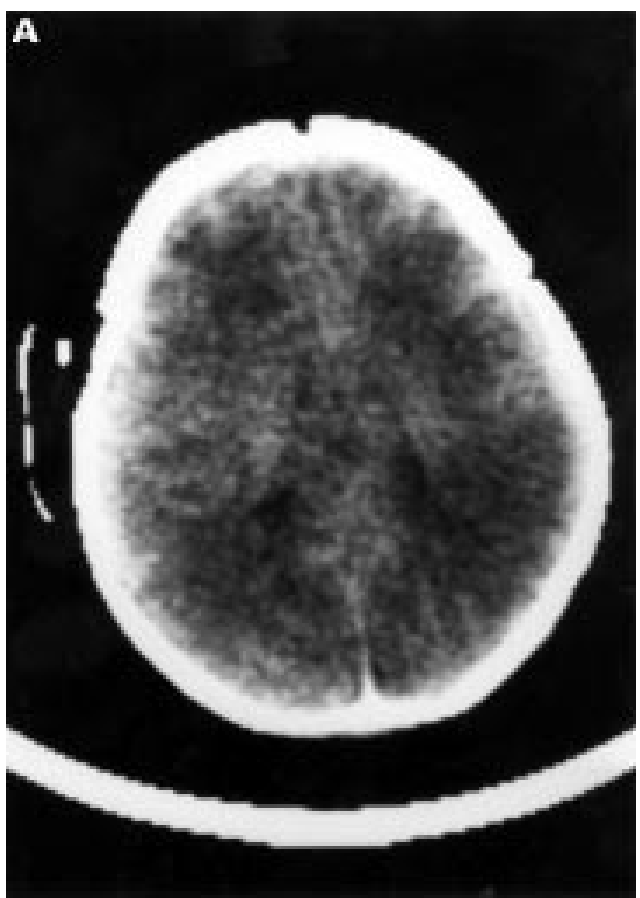

Figure $2 A$ Case 2: boy with a variant of glycogen storage disease type $2 b$. Computed tomogram at 6 days of age shows low density in the cortex and white matter of the parietal and occipital lobes.

occipital lobes (fig 1A). Extensive investigation did not reveal any underlying metabolic cause for the hypoglycaemia.

A magnetic resonance (MRI) examination performed at 10 months of age showed atrophy in the cortex and underlying cerebral white matter, which was most severe in the occipital lobes (fig $1 \mathrm{~B}$ ). At 2 years of age the child was very disabled with spastic quadriplegia, poorly controlled epilepsy, and severe visual impairment.

CASE 2

A boy was delivered at 40 weeks after a normal pregnancy and labour. Apgar scores were 10 at one and five minutes; birthweight was $3.9 \mathrm{~kg}$. The infant breast fed well but developed generalised seizures at 15 hours of age when a blood glucose was measured at $<1.0 \mathrm{mmol} / 1$. Despite intravenous dextrose infusions the child had recurrent severe hypoglycaemic episodes and seizures over the next few days. A computed tomogram on day 6 after birth showed cortical and white matter low attenuation in the parietal and occipital lobes (fig $2 \mathrm{~A}$ ). Biochemical tests and a liver biopsy specimen showed that the hypoglycaemia was caused by a variant of glycogen storage disease type $1 \mathrm{~b}$.

Over the next few years the child was treated with an intensive feeding regimen, including overnight nasogastric feeds, and remained well and seizure free. However, formal testing at the age of 3 years revealed developmental delay. At the age of 7 years he developed complex partial seizures and an MRI scan showed atrophy of the cortex and underlying white matter in the parietal and occipital lobes (fig 2B).

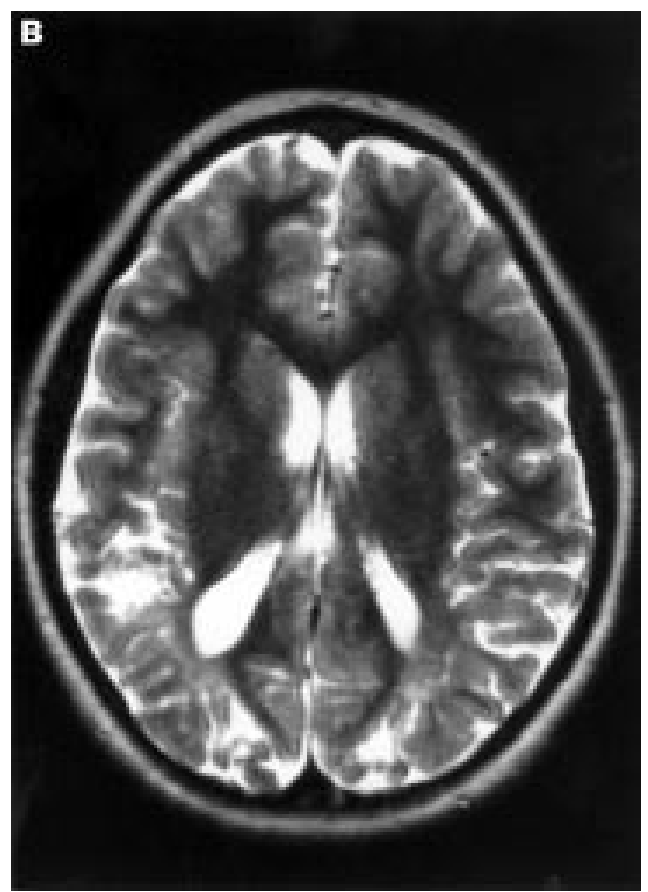

Figure $2 \mathrm{~B} \quad \mathrm{~T}_{2}$ weighted axial magnetic resonance image at 7 years of age shows marked atrophy in the parietal and occipital cortex and underlying cerebral white matter.

\section{Discussion}

Although it is generally accepted that profound neonatal hypoglycaemia is a cause of brain damage ${ }^{34}$ it may be difficult to differentiate the effects of hypoglycaemia from those of secondary seizures and cerebral hypoxiaischaemia. Support for an independent role for hypoglycaemia in causing cerebral injury comes from the finding of different neuropathological changes in hypoglycaemia and ischaemia.

Anderson and colleagues reported the neuropathological findings in neonates in whom hypoglycaemia was regarded as the major cause of death. ${ }^{3}$ In all three cases acute degeneration of neurones and glial cells was present throughout the cerebral cortex but the occipital lobes were the most severely affected. In contrast to the findings in neonatal hypoxiaischaemia, particular involvement of the cortex at the bottom of cerebral sulci (ulegyria) or in vascular "watershed" regions, was not a feature.

The observations of Banker, ${ }^{4}$ based on a study of three hypoglycaemic infants, provide further evidence for a different pattern of cortical damage in neonatal hypoglycaemia and hypoxia-ischaemia. The three cases of hypoglycaemia showed a superficial distribution of damage in the cerebral cortex with particular involvement of layers 2 and 3. In contrast, ischaemia usually affects pyramidal cells in laminae 3 and $5-6 .^{5} \mathrm{~A}$ similar superficial to deep gradient in the density of neuronal necrosis in the cerebral cortex was found in rats exposed to insulin induced hypoglycaemia. ${ }^{5}$

A recent report describes the imaging findings in a case of neonatal hypoglycaemia 
which seem to correlate well with pathological findings. ${ }^{2}$ Both computed tomography and MRI scans revealed extensive cortical loss that was most marked in the occipital regions. We have described very similar imaging findings in two further cases of neonatal hypoglycaemia. These imaging findings differ from those of neonatal hypoxia-ischaemia in term infants where involvement of the parasagittal "watershed" areas is characteristically most evident in the frontal and parieto-occipital regions of the brain. ${ }^{6}$ The difference in pathological findings between neonatal hypoxia-ischaemia and neonatal hypoglycaemia and the predominance of occipital involvement in neonatal hypoglycaemia strongly suggest that hypoglycaemic brain damage is not the result of cerebral hypoxiaischaemia caused by secondary seizures.

1 Hirabayashi S, Kitahara T, Hishida T. Computed tomography in perinatal hypoxic and hypoglycemic encephalopathy with emphasis on follow-up studies. F Comput Assist Tomogr 1980; 4:451-6.

2 Spar J A, Lewine J D, Orrison W W Jr. Neonatal hypoglycemia: CT and MR findings. Am $f$ Neurol Radiol 1994;15:1477-8.

3 Anderson J M, Milner R D G, Strich S J. Effects of neonatal hypoglycemia on the nervous system : a pathological study. f Neurol Neurosurg Psychiatry 1967;30:295-310.

4 Banker B Q. The neuropathological effects of anoxia and hypoglycemia in the newborn. Devel Med Child Neurol 1967;9:544-50

5 Auer R N, Wieloch T, Olsson Y, Siesjö B K. The distribution of hypoglycemic brain damage. Acta Neuropathol (Berl) 1984;64:177-91.

6 Barkovich A J, Truwit C L. Brain damage from perinatal asphyxia: correlation of MR findings with gestational age. Am $\mathcal{f}$ Neurol Radiol 1990;11:1087-96. 